\title{
Performance analysis of adaptive filter channel estimated MIMO OFDM communication system
}

\author{
Srinu Pyla ${ }^{1}$, K Padma Raju ${ }^{2}$, B Bala Subrahmanyam ${ }^{3}$ \\ ${ }^{1,3}$ Department of Electronics and Communication Engineering, G V P College of Engineering (A), India \\ ${ }^{2}$ Department of Electronics and Communication Engineering, J N T University, India
}

\section{Article Info \\ Article history: \\ Received Dec 04, 2017 \\ Revised Feb 16, 2018 \\ Accepted Mar 1, 2018 \\ Keyword: \\ Channel estimation \\ Convolution code \\ LMS \\ MIMO OFDM \\ RLS \\ Space time coding}

\begin{abstract}
Advanced Communication Systems are wideband systems to support multiple applications such as audio, video and data so and so forth. These systems require high spectral efficiency and data rates. In addition, they should provide multipath fading and inter-symbol interference (ISI) free transmission. Multiple input multiple output orthogonal frequency division multiplexing (MIMO OFDM) meets these requirements Hence, MIMOOFDM is the most preferable technique for long term evaluation advanced (LTE-A). The primary objective of this paper is to control bit error rate (BER) by proper channel coding, pilot carriers, adaptive filter channel estimation schemes and space time coding (STC). A combination of any of these schemes results in better BER performance over individual schemes. System performance is analyzed for various digital modulation schemes. In this paper, adaptive filter channel estimated MIMO OFDM system is proposed by integrating channel coding, adaptive filter channel estimation, digital modulation and space time coding. From the simulation results, channel estimated $2 \times 2$ MIMO OFDM system shows superior performance over individual schemes.
\end{abstract}

Copyright (c) 2018 Institute of Advanced Engineering and Science. All rights reserved.

Corresponding Author:

Srinu Pyla,

Department of Electronics and Communication Engineering,

GayatriVidyaParishad College of Engineering (Autonomous),

Madhurawada, Visakhapatnam, Andhra Pradesh, 530048, India.

Email: srinupyla@gvpce.ac.in

\section{INTRODUCTION}

Advanced mobile systems aim on supporting high data rate services over multiple systems for multimedia applications involving voice, data, pictures and video. They should provide services without interruptions for private and public networks. These services require interference free transmission over frequency selective fading channels. OFDM is a promising technique for Long Term Evaluation-Advanced (LTE-A), Worldwide Interoperability for Microwave Access (Wi-MAX) systems, IEEE 802.11ac, and IEEE 802.15 [1]. A good channel estimation procedure is necessary in order to make reliable decisions and to coherently demodulate the received data. OFDM systems require effective and exact channel estimation for time-varying channels.

The principal motto of this paper is to improve the OFDM system performance by proper channel coding, suitable digital modulation schemes, adaptive filter channel estimation techniques and multiple antennas at the transmitter and receiver. By using all these, an integrated OFDM system is proposed to enhance system performance [2-3]. Gosh [4] formulated a closed form expression for impulse noise performance in a multicarrier QAM system and showed that it is better than single carrier systems for moderate values of impulse power and probability. Dong et al. [5] determined the power and rate adaptation analytically with channel state information in the presence of carrier frequency offset (CFO) for an OFDM system. Sabbaghian et al. [6] developed a new code based on dual BCH code for reduction of PAPR in multi 
carrier system. Hong yu et al. [7] developed an iterative based ML algorithm for multiple solution complication from log likelihood function in OFDM mobile wireless channels. Kalyani et al. [8] proposed a channel frequency response estimation algorithm with limited number of pilots to determine the selectivity of the channel in an OFDM system. Han oh et al. [9] described an estimation scheme to detect symbols which are determined by the adaptive threshold using the noise variance with the different posteriori probability at each sub-carrier. Gao et al. [10] developed adaptive frequency domain channel estimation for uplink in MIMO-OFDM system to improve channel estimation by making use of correlation between two sub-carriers. Souryal et al. [11] reported that the sub-channel bit and power can be allocated dynamically to the state of the channel rather than fixed and uniform modulation. It was shown that bit allocation based on the prediction state of the channel decreases the BER by a factor of two with a maximum Doppler frequency of $50-100$ $\mathrm{Hz}$. The performance of adaptive OFDM over non adaptive OFDM is good, even in the presence of channel estimation errors for slowly varying channels.

Hong et al. [12] proposed adaptive all pass filter scheme in which phase rotation is used to reduce PAPR in MIMO-OFDM systems. Wang et al. [13] modeled adaptive receiver that is used for reduction of inter carrier interference with the help of optimal phase rotation. Elangoven et al. [14] described a pilot channel estimation in which fading effects in the channel are equalized by using LMS, NLMS and RLS algorithms. A Salh et al. [15] described the achievement of higher data rates through zero forcing beam forming with less number of radio frequency chains. Erik G. Larsson et al. [16] stated that the spatial multiplexing is used in Massive MIMO to increase the capacity, nearly ten times or more and improve the radiated energy in the order of hundred times simultaneously. Bolcskei et al. [17] described the fundamentals of MIMO-OFDM wireless systems and hardware implementation aspects of fourth generation cellular systems. Stuber et al. [18] discussed issues related to synchronization and channel estimation in MIMOOFDM along with space coding techniques. In [19], serial concatenated convolution code for MIMO systems are described under Rayleigh fading channels.

\section{SYSTEM MODEL}

Let $\mathrm{N}$ be the number of subcarriers in an OFDM symbol, then the baseband discrete time OFDM signal sequence can be written as

$$
x(n)=\operatorname{IFFT}(X(K))=\frac{1}{N} \sum_{k=0}^{N-1} X(K) e^{\frac{j 2 \pi k n}{N}}
$$

Where, $n=0,1,2, \ldots N-1$ and $K$ is the index of the sub-carrier. Before transmission, cyclic prefix (CP) is appended periodically to prevent $\mathrm{x}_{\mathrm{g}}(\mathrm{n})$ from ISI. The resultant OFDM symbol is transmitted through frequency selective fading channel $\mathrm{h}(\mathrm{n})$. Noise is added to the receiving signal in the channel. The received signal is expressed as

$$
y_{g}(n)=x_{g}(n) * h(n)+v(n)
$$

Where $v(n)$ is the additive white Gaussian noise. At receiver, serial data is converted into parallel and CP is removed from $\mathrm{y}_{\mathrm{g}}(\mathrm{n})$ to form $\mathrm{y}(\mathrm{n})$ and is expressed as

$$
Y(K)=\operatorname{FFT}\{y(n)\}=\sum_{n=0}^{N-1} y(n) e^{-\frac{j 2 \pi n k}{N}}
$$

where $k=0,1,2, \ldots N-1$. Further, $\mathrm{Y}(\mathrm{K})$ can be written as

$$
\mathrm{Y}(\mathrm{K})=\mathrm{X}(\mathrm{K}) \mathrm{H}(\mathrm{K})+\mathrm{V}(\mathrm{K}) \quad \mathrm{k}=0,1, . . \mathrm{N}-1
$$

Pilots are extracted from the obtained sequence $\mathrm{Y}(\mathrm{K})$. The estimated output $\mathrm{X}_{\mathrm{e}}(\mathrm{K})$ is obtained by:

$$
X_{e}(K)=\frac{Y(K)}{H(K)}
$$

Transmitted data is recovered at the receiver by using proper demodulator. 


\begin{tabular}{llll} 
& \multicolumn{2}{c}{ Table 1. Parameters used in the Proposed System } \\
\hline Parameter & Specification & Parameter & Specification \\
\hline $\begin{array}{l}\text { Number of sub- } \\
\text { carriers }\end{array}$ & 64 & Coding & Convolution \\
FFT size & 64 & Code rate & $1 / 2,1 / 3$ \\
Guard type & Cyclic extension & Wireless channels & Rayleigh, Rician and AWGN \\
Cyclic prefix & $16(1 / 4)$ & Channel estimation & LMS, RLS \\
Modulation schemes & BPSK, QPSK, 16- & Antenna configurations & SISO, 2×1 MISO, 2×2 MIMO \\
\hline
\end{tabular}

\subsection{Characteristics of wireless channel}

In real time applications, the data transmission is much more complex due to many factors such as line of sight communication between transmitter and receiver, user's density, multipath propagation, Doppler shift and so on and so forth. Hence, different channel models such as additive white Gaussian noise, Rayleigh and Rician channels are formulated. AWGN channel model is the most commonly used channels in communication systems. The density function for Gaussian distribution is represented in [20] as

$$
p(x)=\frac{1}{\sigma \sqrt{ } 2 \Pi} \exp \left(-\frac{(x-\mu)^{2}}{2 \sigma^{2}}\right) ;-\propto<x<\propto
$$

However, in this channel, multipath fading, frequency selectivity and interference are not considered and moreover, it is not suitable for terrestrial applications. Rayleigh and Rician channel models are developed for multipath propagation applications. The received signal is distorted due to attenuation, reflections, refraction, and diffraction during transmission. The statistical time varying nature of received signal power is described using Rayleigh and Rician fading distributions. The envelope of received flat faded OFDM signal in non-line of sight (NLOS) scenario resembles Rayleigh fading distribution whose probability density function (PDF) is characterized in [20] by

$$
p(x)=\frac{x}{\sigma^{2}} \exp \left(-\frac{x^{2}}{2 \sigma^{2}}\right) \sigma>0, x \geq 0
$$

When a strong signal component exists in addition to multiple weak reflected components, the received signal envelope is in the form of Rician distribution [16] and its probability distribution function is expressed in [20] as

$$
\begin{aligned}
& p(x)=\frac{x}{\sigma^{2}} \exp \left(-\frac{\left(x^{2}+A^{2}\right)}{2 \sigma^{2}}\right) I_{0}\left(\frac{A x}{\sigma^{2}}\right) \\
& \text { for } x \geq 0 ; A \geq 0
\end{aligned}
$$

Where $I_{0}$ is the modified Bessel function of the first kind and zero ${ }^{\text {th }}$ order and $\mu$ is the mean due to fixed scatter or LOS path. K-factor of Rician distribution is the square of the peak amplitude of the dominant signal to twice the variance. K-factor is typically in between 1 and 10 and Rician distribution approaches Rayleigh whenever $\mathrm{K}$ tends to zero

\subsection{Channel coding}

The aim of the channel coding is to enhance the reliability of the communication system through improving bit error rate (BER) performance by appending redundant bits to the transmitted data. These additional bits are used to make the system capable of error detection and correction. In this work, convolution code with code rates $1 / 2$ and $1 / 3$ is considered for encoding the data. Convolution coding has memory that uses previous bits to encode or decode the input data stream. Figure 1 , the convolution encoder has three variables: number of input bits ' $k$ ', output bits ' $n$ ' and memory block length ' $L$ '. The received bits can be decoded using a soft input-hard output Viterbi decoding algorithm. Soft input decision action is taken based on whole input range values while hard output decision action gives decoded bits based on fixed set of values. Viterbi algorithm uses code trellis and minimum hamming distance.

\subsection{Channel estimation techniques}

The subcarriers in OFDM system exhibit their own characteristics during the transmission. Hence, adaptive channel estimation is suitable for proper recovery of the transmitted signal at the receiver. Channel properties can be estimated using pilot based approaches. Block and comb type pilot arrangements are suitable for slow and fast fading channels respectively. In block type pilot arrangement, pilots are inserted in specific OFDM blocks periodically whereas in comb type, pilots are inserted uniformly in every OFDM 
block [21]. Popular channel estimation schemes are Least Squares (LS), Minimum Mean Square Error (MMSE), Least Mean Squares (LMS), and Recursive Least Squares (RLS). Pilot arrangements for channel estimation as shown in Figure 2.

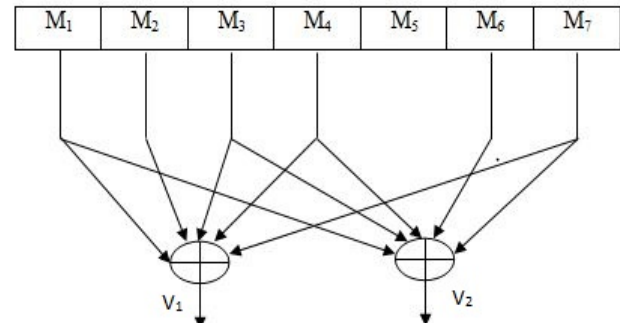

Figure 1. Convolution encoder with code rate 1/2.

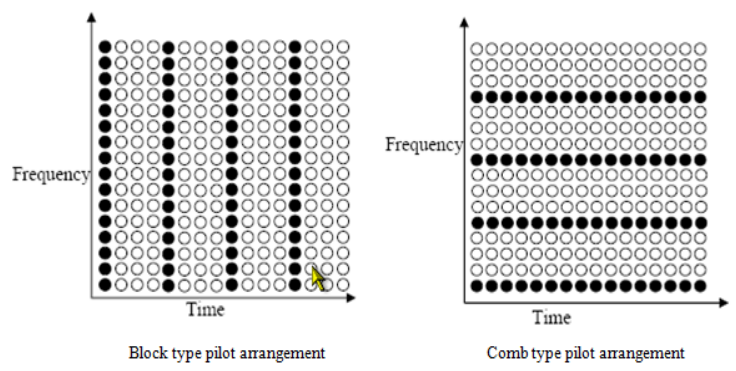

Figure 2. Pilot arrangements for channel estimation

\subsubsection{Linear channel estimation schemes:}

The received signal can be expressed as

$$
\boldsymbol{Y}=\boldsymbol{X} \boldsymbol{H}+\boldsymbol{V}
$$

where $\mathbf{X}$ and $\mathbf{Y}$ are the transmitted and received signal vectors respectively. Here, $\mathbf{H}$ is the channel response while $\mathbf{V}$ is the noise vector. The LS estimator for an OFDM system can be written as

$$
\begin{aligned}
& H_{L S}=\left(X^{H} X\right)^{-1} X^{H} Y \\
& H_{L S}=X^{-1} Y
\end{aligned}
$$

The estimate of the channel at $\mathrm{m}^{\text {th }}$ pilot subcarrier is given by

$$
H_{p}(m)=\frac{Y_{p}(m)}{X_{p}(m)}
$$

The mean square error from the received signal can be described as

$$
\begin{aligned}
& J(e)=E\left[(H-\widehat{H})^{2}\right] \\
& J(e)=E\left[(H-\widehat{H})^{H}(H-\widehat{H})\right]
\end{aligned}
$$

where ' $\widehat{H}$ ' is the MMSE channel estimate and $\mathrm{X}^{\mathrm{H}}$ is the Hermitian matrix of $\mathrm{X}$. MMSE equaliser sets orthogonal by estimating vector $\mathrm{Y}$ as:

$$
\begin{aligned}
& E\left[(H-\widehat{H}) Y^{H}\right]=0 \\
& E\left[H Y^{H}\right]-E\left[Y Y^{H}\right]=0 \\
& R_{h Y}-R_{Y Y}=0
\end{aligned}
$$

The time domain MMSE estimate of ' $h$ ' is represented as

$$
\hat{h}=R_{h Y} R_{Y Y}^{-1} Y
$$

\subsubsection{Adaptive filter channel estimation schemes:}

The LMS algorithm is a gradient-based procedure of steepest-descent that adapts the iterative procedure in which successive weight vectors are modified to obtain MMSE. The LMS estimator uses adaptive filter as shown in Figure 3. The initial weight is assumed and the successive values are computed based on previous estimation. The LMS algorithm iterates over each coefficient in the direction of approximate gradient. The weight vector equation is expressed as:

$$
w(n+1)=w(n)+\mu[e(n) x(n)]
$$


where $\mu$ is the step size, $L$ is the number of iterations, e(n) is the error between received signal $y(n)$ and reference signal $d(n)$. Here, $x(n)$ is the input signal to the LMS filter. The minimum mean square error is of the form

$$
e^{2}(n)=[d(n)-y(n)]^{2}
$$

The corrected received signal at the output of LMS filter can be shown as

$$
y(n)=w(n) * x(n)
$$

The LMS estimate has lower computational complexity. However, it has poor tracking ability with slower convergence rate. In the RLS algorithm, previous samples are used to estimate the inverse of autocorrelation of input vector. The RLS algorithm uses past input data samples to estimate the inverse of the autocorrelation matrix of the input vector. A weighting factor for each sample is used to decrease the influence of very old input samples. The RLS estimator uses adaptive filter as shown in Figure 4.

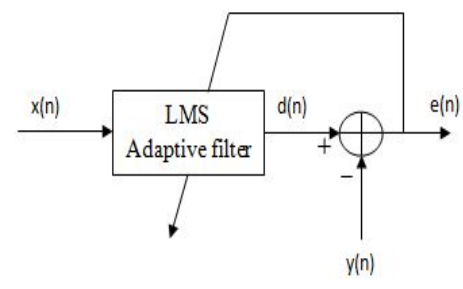

Figure 3. LMS Adaptive Filter

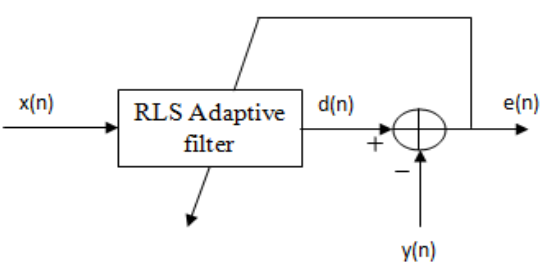

Figure 4. RLS Adaptive Filter

The RLS algorithm needs auto-correlation and cross-correlation matrix of input and is significantly more computationally complex while updating each coefficient, since it needs inverse of the correlation matrix $\phi(n)$. However, the RLS channel estimation algorithm has faster convergence with excellent tracking ability compared to LMS algorithm. RLS algorithm is described as;

$$
\begin{aligned}
& P(n)=\emptyset^{-1}(n) \\
& k(n)=\frac{\lambda^{-1} P(n-1) x(n)}{1+\lambda^{-1} x^{H}(n) P(n-1) x(n)} \\
& e(n)=d(n)-y(n) \\
& w(n+1)=w(n)+k(n) e(n)
\end{aligned}
$$

Here, $\lambda$ is the forgetting factor and for $0<\lambda<1$. The influence of past error values decreases exponentially and it is termed as 'method of exponentially weighted least squares' [14].

\subsubsection{MIMO-OFDM}

OFDM transforms a wideband frequency selective channel into a number of flat fading channels which is suitable for MIMO structure that requires a frequency non-selective characteristic at each channel when the transmission rate is high. The MIMO is in the form of $M_{T} \times M_{R}$, where $M_{T}$ is the number of transmitting antennas and $M_{R}$ is the number of receiving antennas. Let $M_{T}=2$ and $M_{R}=2$, then the MIMO channel matrix ' $\mathrm{H}$ ' can be expressed as

$$
\mathrm{H}=\left[\begin{array}{ll}
h_{11} & h_{12} \\
h_{21} & h_{22}
\end{array}\right]
$$

Here, ' $h_{i j}$ ' is the fading coefficient that indicates the impulse response between $\mathrm{i}^{\text {th }}$ transmit antenna and $\mathrm{j}^{\text {th }}$ receiving antenna. The MIMO system model is modeled as

$$
\begin{aligned}
& Y=H X \\
& {\left[\begin{array}{l}
y_{1} \\
y_{2}
\end{array}\right]=\left[\begin{array}{ll}
h_{11} & h_{12} \\
h_{21} & h_{22}
\end{array}\right] \times\left[\begin{array}{l}
x_{1} \\
x_{2}
\end{array}\right]}
\end{aligned}
$$


MIMO is known as 'spatial multiplexing' as the received signal samples through all possible paths from multiple transmitting antennas are combined by the spatial ratio combiner at the receiver. This multiple transmit-receive antenna pair offers a linear increase in transmission rate for the same bandwidth without any additional power required. It can also increase diversity gain by transmitting the information signal through multiple antennas using space time coding. Alamouti scheme is the basic space time code for MIMO system is described in (27)

$$
S=\left[\begin{array}{cc}
S_{1} & S_{2} \\
-S_{2}^{*} & S_{1}^{*}
\end{array}\right]
$$

where $\mathrm{S}_{1}{ }^{*}$ is the complex conjugate of $\mathrm{S}_{1}$ symbol and $\mathrm{S}_{2}{ }^{*}$ is the complex conjugate of $\mathrm{S}_{2}$ symbol. The encoder outputs are transmitted in two consecutive time slots from two transmitting antennas. In first time slot, $S_{1}$ and $\mathrm{S}_{2}$ are transmitted and in the consecutive time slot $-\mathrm{S}_{2}{ }^{*}$ and $\mathrm{S}_{1}{ }^{*}$ are transmitted. Received signals at first and second time slots are $\mathrm{y}(1)$ and $\mathrm{y}(2)$ respectively can be written as

$$
\begin{aligned}
& y(1)=\left[\begin{array}{ll}
h_{1} & h_{2}
\end{array}\right]\left[\begin{array}{l}
S_{1} \\
S_{2}
\end{array}\right]+n(1) \\
& y(2)=\left[\begin{array}{ll}
h_{1} & h_{2}
\end{array}\right]\left[\begin{array}{c}
-S_{2}^{*} \\
S_{1}^{*}
\end{array}\right]+n(2)
\end{aligned}
$$

where $h_{1}$ and $h_{2}$ are channel responses in the $1^{\text {st }}$ and $2^{\text {nd }}$ path respectively, and $n(1)$ and $n(2)$ are additive white Gaussian noises at first and second time slots respectively. Hence, the MIMO system model can be described:

$$
\left[\begin{array}{l}
y(1) \\
y(2)
\end{array}\right]=\left[\begin{array}{cc}
h_{1} & h_{2} \\
h_{2}^{*} & -h_{1}^{*}
\end{array}\right]+\left[\begin{array}{l}
n(1) \\
n(2)
\end{array}\right]
$$

The column channel matrices $\mathrm{C}_{1}$ and $\mathrm{C}_{2}$ are represented as:

$$
C_{1}=\left[\begin{array}{l}
h_{1} \\
h_{2}^{*}
\end{array}\right] \text { and } C_{2}=\left[\begin{array}{c}
h_{2} \\
-h_{1}^{*}
\end{array}\right]
$$

The inner product of two columns $\mathrm{C}_{1}$ and $\mathrm{C}_{2}$ becomes zero as shown in equation (31).

$$
C_{1}^{H} C_{2}=h_{1}^{*} h_{2}+h_{2}\left(-h_{1}^{*}\right)=0
$$

In equation (31) confirms the orthogonality of Alamouti scheme. The capacity and error rate analysis of MIMO communication system for satellite applications are well described in [22]. The capacity of MIMO channel is given in (32)

$$
C=\log _{2} \operatorname{det}\left(I_{N_{R}}+\frac{S N R}{N_{T}} H H^{H}\right)
$$

where, $I_{N_{R}}$ is $N_{R}$ x $N_{R}$ identity matrix, $H$ is the channel matrix, and $N_{T}$ is the number of transmitting antennas.

\section{PROPOSED METHOD}

The proposed MIMO OFDM system is shown in Figure 5. In the proposed system, information data are encoded using a convolution encoder with code rates $1 / 2$ and $1 / 3$. The encoded data are applied to digital modulator in order to convert data into symbols and then pilot symbols are inserted for channel estimation. Then IFFT converts these symbols into time domain and makes the symbols orthogonal to one another. Cyclic prefix (CP) is appended to the output of IFFT to prevent the signal from ISI. In general, the cyclic prefix is taken from the tail of the previous OFDM symbol. The CP length should be greater than the channel response in order to remove ISI completely. After the insertion of CP, the OFDM signal is applied to the space time block code (STBC) encoder for transmission of signal via multiple antennas. CP is removed at the receiver and channel is estimated using either LMS or RLS algorithm. The reverse process of the transmitter is applied in order to recover the transmitted information. 


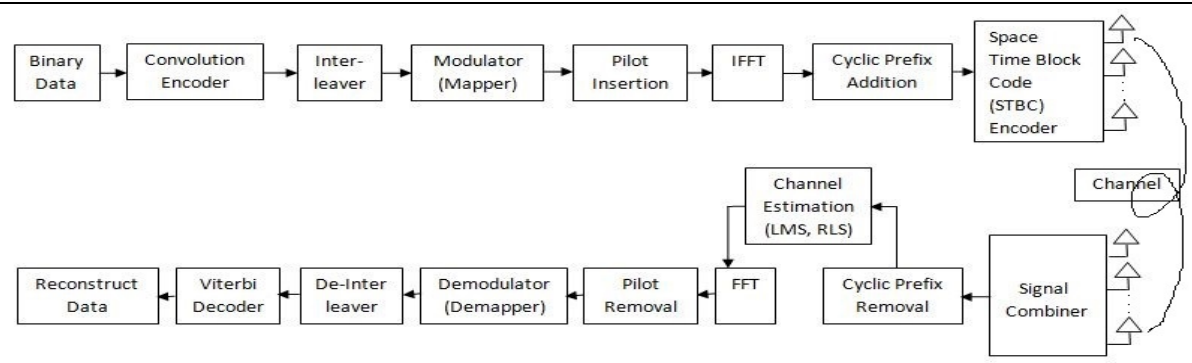

Figure 5. Integrated MIMO OFDM system

\section{RESULTS AND ANALYSIS}

The proposed MIMO-OFDM system is implemented with the parameters mentioned in Table 1. In this paper, the OFDM system performance is analyzed for various channels as shown in Figure 6 and it illustrates the better BER performance of additive white Gaussian channel in comparison with the Rayleigh and Rician channels. Figure 7 shows the performance of OFDM system for various modulation schemes under Rician channel and it describes the performance improvement with decrease in order of modulation. Figure 7 and Figure 8 shows approximately 2 dB SNR improvement in LMS algorithm over RLS algorithm at 10-3BER under BPSK modulation. Better performance of BPSK modulation over QPSK modulation can be observed from Figure 9 to Figure10. The performance of convolution code with different code rates is analyzed in Figure 11 and Figure 12 which illustrates the improvement of system performance with decrease in code rate. Performance improvement of MIMO can be observed from Figure 12 and Figure 13. Hence, the proposed 2x2 MIMO OFDM system shows superior performance as depicted in Figure 14 over other combinations. Moreover, the proposed system performance is better over the system [23] in which the performance of MIMO-OFDM in terms of bit error rate is discussed for LS and MMSE channel estimation schemes. In addition, this system results superior performance over [24] in which the BER performance of MIMO-OFDM system for NLMS and DD-EW RLS channel estimation schemes is analyzed. Performance comparison of $2 \times 2$ MIMO OFDM system for digital modulation schemes under Rayleigh and Rician channels as shown in Figure 15. Figure 16 shows the system capacity improvement with order of MIMO.

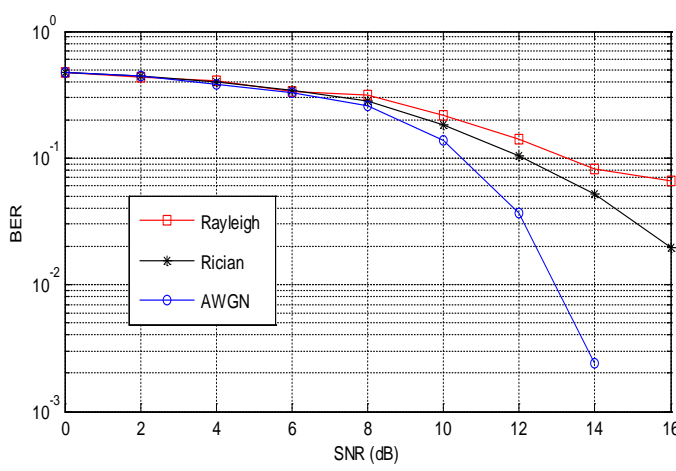

Figure 6. Performance of 16-QAM OFDM under fading6 channels

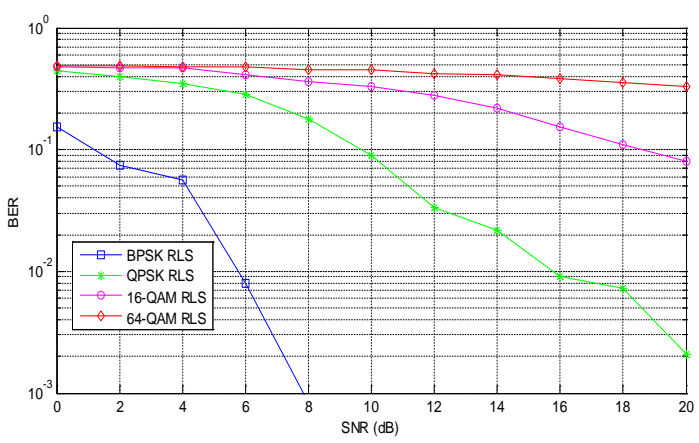

Figure 8. BER performance comparison of convolution coded OFDM using RLS

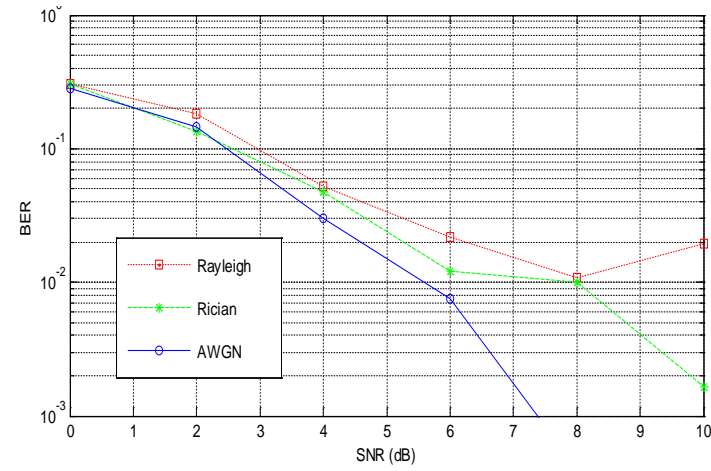

Figure 7. Performance of BPSK OFDM under fading channels

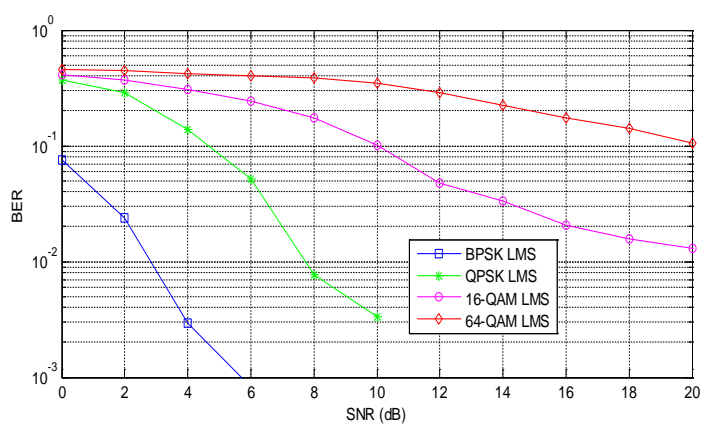

Figure 9. BER performance comparison of convolution coded OFDM using LMS

Performance analysis of adaptive filter channel estimated MIMO OFDM communication... (Srinu Pyla) 


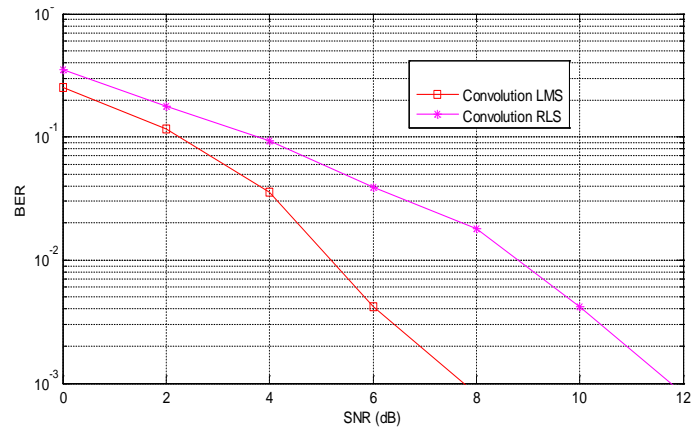

Figure 10. Performance of OFDM with 1/2 rate convolution code, LMS, RLS, BPSK modulation

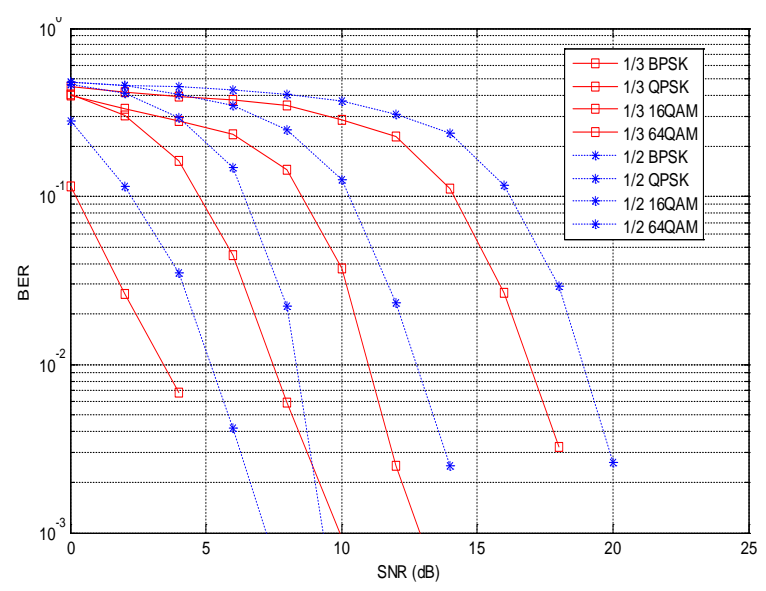

Figure 12. OFDM system performance comparison for different modulation schemes and code rates

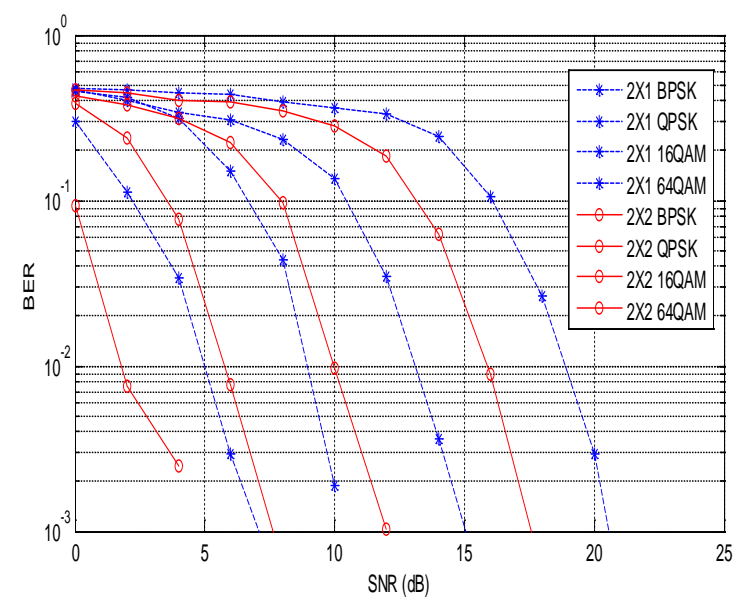

Figure 14. Performance comparison of $2 \times 1$ and $2 \times 2$ MIMO OFDM systems for digital modulation schemes

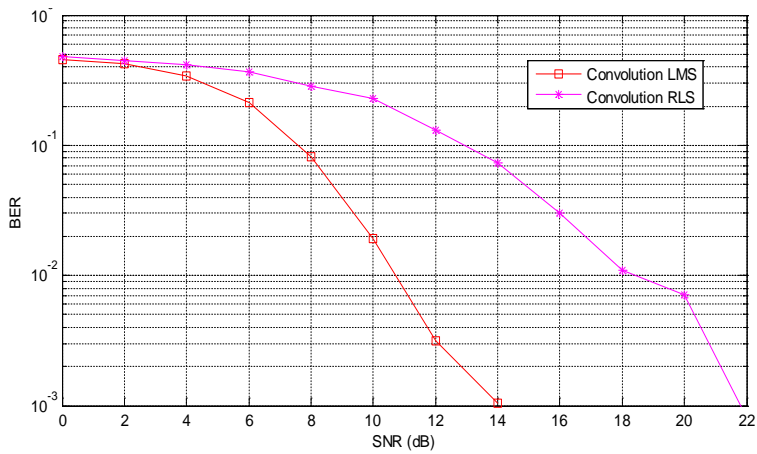

Figure 11. Performance of OFDM with $1 / 2$ rate convolution code, LMS, RLS, QPSK modulation

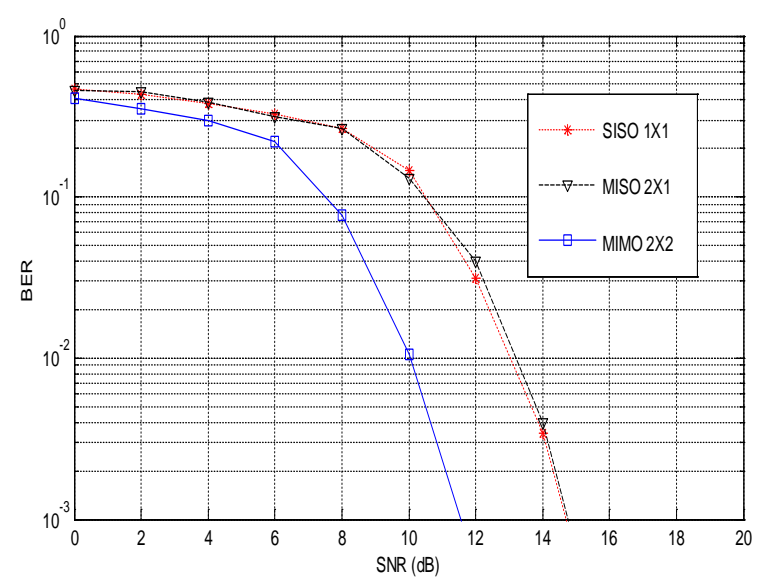

Figure 13. Performance comparison of SISO, $2 \times 1$ and $2 \times 2$ MIMO OFDM systems.

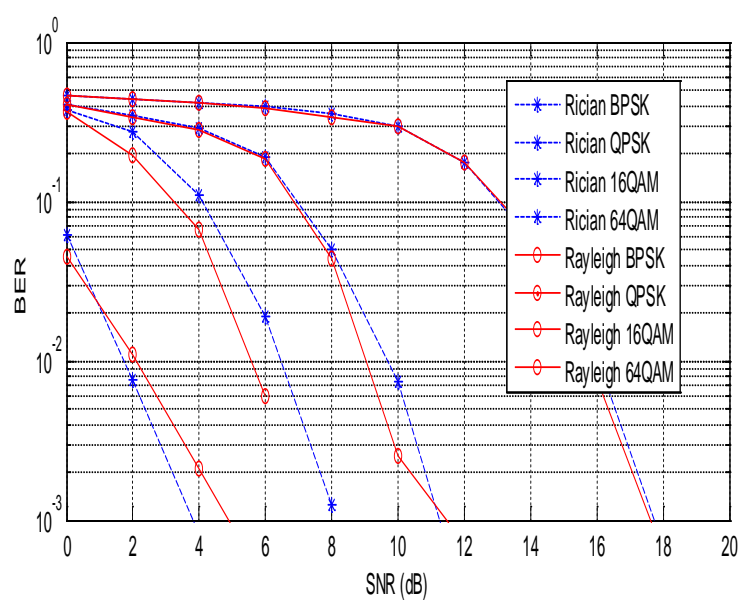

Figure 15. Performance comparison of $2 \times 2$ MIMO OFDM system for digital modulation schemes under Rayleigh and Rician channels. 


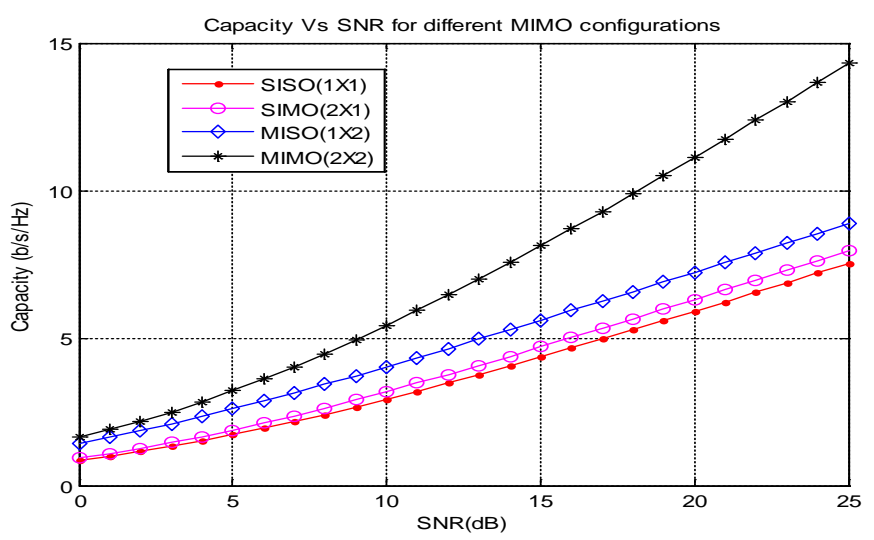

Figure 16. Capacity improvement with increase in MIMO configuration for MIMO OFDM system

\section{CONCLUSION}

In the proposed work, the MIMO-OFDM system is simulated with 64 sub carriers, digital modulation techniques (BPSK, QPSK, 16-QAM and 64-QAM), convolution code with code rates (1/2 and 1/3), channels (AWGN, Rayleigh and Rician), adaptive filter channel estimation schemes (LMS and RLS) and MIMO configurations (SISO, 2×1 MIMO and 2×2 MIMO). From the results, it is observed that the BER performance of AWGN channel is superior compared to Rayleigh and Rician and also spectral efficiency increases with order of modulation with marginal degradation in performance. There is an improvement in system performance with decrease in code rate and the LMS channel estimation is found to be better than the RLS channel estimation. By combining channel estimation and coding, there is further improvement in OFDM system performance over individual schemes. 2x2 MIMO OFDM system shows improved performance over SISO and 2x1 MISO. Moreover, an increase in the order of MIMO configuration increases capacity of the MIMO system. Finally, the proposed channel estimated $2 \times 2$ MIMO OFDM system shows significant improvement in BER performance over other combinations.

\section{REFERENCES}

[1] Aleksandra Panajotovi, FelipRiera-Palou and GuillemFemenias, "Adaptive Uniform Channel Decomposition in MU-MIMO-OFDM: Application to IEEE 802.11ac,” IEEE transactions on wireless communications, 1536-1276, Vol.14, No.5, May 2015.

[2] Ahmed Temtam, Dimitrie C. Popescu, and OtiliaPopescu, "Using OFDM Pilot Tone Information to Detect Active 4G LTE Transmissions,” IEEE transactions on wireless communications, 2014.

[3] Md. MahmudulHasan, Saleh Mohammad Sagar, "Wireless Communication through Long Term Evolution (LTE) over Satellite Channel by using MIMO-OFDM Model,” ICAEE, 19-21, Dec 2013.

[4] M. Ghosh, "Analysis of the effect of impulse noise on multicarrier and single carrier QAM systems," IEEE Transactions on Communications, vol. 44, no. 2, pp. 145-147, Feb. 1996.

[5] Zhicheng Dong, Pingzhi Fan, ErdalPanayirci and Xianfu Lei, "Conditional Power and Rate Adaptation for MQAM/OFDM Systems under CFO with Perfect and Imperfect Channel Estimation Errors,” IEEE Transactions on Vehicular Technology, Vol. 64, No. 11, Nov 2015.

[6] Maryam Sabbaghian, YongjunKwak and VahidTarokh, "New Codes from Dual BCH Codes with Applications in Low PAPR OFDM,” IEEE transactions on Wireless communications, Vol. 10, 1536-1276, No.12, Dec 2011.

[7] Hong-Yu Liu and R. Y. Yen, "Effective Adaptive Iteration Algorithm for Frequency Tracking and Channel Estimation in OFDM Systems,” IEEE transactions on Vehicular technology, vol. 59, 0018-9545, No. 4, May 2010.

[8] SheetalKalyani, RaghavendranLakshminarayanan, Krishnamurthy Giridhar, "Biased estimators with adaptive shrinkage targets for orthogonal frequency division multiple access channel estimation,” IET Commun., 2013, Vol. 7, Iss. 1, pp.13-22, 2013.

[9] Jun-Han Oh and Jong-Tae Lim, “Two-Step Channel Estimation Scheme for OFDM Systems over Fast Rayleigh Fading Channels,” IEEE Communications letters, vol.14, No.6, 1089-7798, June 2010.

[10] J. Gao X. Zhu Y. Wu A.K. Nandi, "Kalman smoothing-based adaptive frequency domain channel estimation for uplink multiple-input multiple-output orthogonal frequency division multiple access systems," IET Communications, Vol. 5, Iss. 2, pp. 199-208, 2011.

[11] M. R. Souryal and Raymond L. Pickholtz, “Adaptive Modulation with Imperfect Channel Information in OFDM,” IEEE journal, 2001.

[12] Eonpyo Hong and DongsooHar, "Peak-to-Average Power Ratio Reduction for MISO OFDM Systems with Adaptive All-Pass Filters," IEEE transactions on Wireless communications, vol.10, 1536-1276, No.10, October 2011. 
[13] Chin-Liang Wang, Po-Chung Shen, Ying-Chang Lin, and Jia-Hong Huang, “An Adaptive Receiver Design for OFDM Systems Using Conjugate Transmission,” IEEE transactions on communications, vol.61, 0090-6778, No.2, Feb 2013.

[14] K. Elangovan, "Comparative study on the channel estimation for OFDM system using LMS, NLMS and RLS algorithms,” International conference on Pattern Recognition, Informatics and Medical engineering, pp. 359-363, March 21-23, 2012.

[15] A Salh, L Audah, N S M Shah and S A Hamzah, "Asaptive antenna selection and power allocation in downlink massive MIMO systems," international journal of electrical and computer engineering (IJECE), vol. 7, no. 6, pp. 3521-3528, December 2017.

[16] Erik G.Larsson, OveEdfors, Fredrik Tufvesson and Thomas L. Marzetta "Massive MIMO for next generation wireless systems,” IEEE Communication Magazine, 186-195, Feb 2014.

[17] H. Bolcskei, Eth Zurich "MIMO-OFDM wireless systems: basics, perspectives and challenges,” IEEE Wireless Comm., 31-37, Aug 2006.

[18] Gordon L.Stuber, John R.Barry, Steve W.Mclaughlin, YE LI, Mary Ann Ingram "Broadband MIMO-OFDM wireless communications,” Proceedings of IEEE, Vol.92, No.2, 271-294, Feb 2004.

[19] Ilesanmi Banjo Oluwafemi, "Hybrid concatenated coding scheme for MIMO systems," international journal of electrical and computer engineering (IJECE), vol. 5, no. 3, pp. 464-476, June 2015

[20] Theodore S. Rappaport, "Wireless Communications-Principles and Practice," Pearson Publications, Second edition, 2011.

[21] SrinuPyla, Padma Raju K, and Balasubrahmanyam N, "Performance Analysis of OFDM system using pilots, coding bounds and MAP decoder for next generation applications," International Journal on Communications Antenna and Propagation (IRECAP), 2017.

[22] RamoniAdeogun, "Capacity and error rate analysis of MIMO satellite communication systems in fading scenarios,” international journal of electrical and computer engineering (IJECE), vol. 4, no. 4, pp. 614-622, August 2014.

[23] P Venkateswarlu, and R. Nagendra, "Channel estimation in MIMO-OFDM systems,” international journal of engineering trends and technology, vol. 15, no. 5, Sep 2014.

[24] S. S. Sarnin and S. M. Sulong, "Performance of channel estimation based on DD-EW RLS and NLMS for outdoor MIMO OFDM system,” proceedings of ICSPC-2014, pp. 90-95, Dec 2014. 